\title{
Dynamical systems analysis of fluid transport in time-periodic vortex ring flows
}

\author{
Karim Shariffa) \\ NASA Ames Research Center, Moffett Field, California 94035 \\ Anthony Leonard \\ California Institute of Technology, Pasadena, California 91125 \\ Joel H. Ferziger \\ Stanford University, Stanford, California 94305
}

(Received 18 October 2005; accepted 28 February 2006; published online 17 April 2006)

\begin{abstract}
It is known that the stable and unstable manifolds of dynamical systems theory provide a powerful tool for understanding Lagrangian aspects of time-periodic flows. In this work we consider two time-periodic vortex ring flows. The first is a vortex ring with an elliptical core. The manifolds provide information about entrainment and detrainment of irrotational fluid into and out of the volume transported with the ring. The likeness of the manifolds with features observed in flow visualization experiments of turbulent vortex rings suggests that a similar process might be at play. However, what precise modes of unsteadiness are responsible for stirring in a turbulent vortex ring is left as an open question. The second situation is that of two leapfrogging rings. The unstable manifold shows striking agreement with even the fine features of smoke visualization photographs, suggesting that fluid elements in the vicinity of the manifold are drawn out along it and begin to reveal its structure. We suggest that interpretations of these photographs that argue for complex vorticity dynamics ought to be reconsidered. Recently, theoretical and computational tools have been developed to locate structures analogous to stable and unstable manifolds in aperiodic, or finite-time systems. The usefulness of these analogs is demonstrated, using vortex ring flows as an example, in the paper by Shadden, Dabiri, and Marsden [Phys. Fluids 18, 047105 (2006)].

(C) 2006 American Institute of Physics. [DOI: 10.1063/1.2189867]
\end{abstract}

\section{INTRODUCTION}

In the 1980 s, considerable effort, under the rubric of "chaotic advection" or "Lagrangian turbulence," was devoted to applying dynamical systems theory concepts, such as the Poincaré section and Lyapunov exponent, to study the transport of fluid elements in time-periodic flows. Aref ${ }^{1}$ demonstrated some ideas through a toy model of stirring in a cylindrical enclosure consisting of two vortices that are alternately turned on and off. This was followed by numerical ${ }^{2}$ and experimental ${ }^{3,4}$ studies in Stokes flows, for instance the flow in the space between two nonconcentric cylinders. The first to consider an open flow were Rom-Kedar et al., ${ }^{5}$ who introduced the oscillating vortex pair model in which the positions of a counter-rotating pair of point vortices are subject to oscillatory forcing. They showed that the stable and unstable manifolds of the fixed points of the Poincare map play an important role. In particular, they provide a template for the flow, and lobes formed by intersections of the two manifolds can be used to quantify rates of entrainment and detrainment into the volume of fluid transported with the vortex pair. They showed that the areas of intersection of certain lobes can be used to predict the decay of the average concentration of a tracer initially placed in the oval surrounding the vortex pair. Solomon et l. $^{6}$ experimentally created a

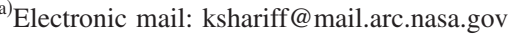

flow with a row of many vortices (alternating in sign) enclosed in a channel. They showed that the lobe area can be used to calculate a diffusion coefficient that agrees with an independent determination based on the dispersion of dye.

The present work, which was also performed in the 1980s, follows upon the heels of Rom-Kedar et al. and computes manifolds for time-periodic vortex ring flows. We take up the suggestion that instead of externally imposing the period and amplitude of the unsteadiness, "Future studies might focus on the dynamics of flows specified by an internal parameter...in which changes in the parameter result in changes in flow kinematics and thus the mixing." end, we consider solutions of the Euler equations that are inherently unsteady with period and amplitude determined internally. Two cases are considered. The first is that of a vortex ring having a core of (practically) uniform vorticity and elliptic cross section, the solution for which was obtained by Moore. ${ }^{8}$ The core rotates at the constant angular velocity of Kirchhoff's elliptical vortex (Lamb, ${ }^{9}$ Sec. 159) and its speed of translation oscillates about the value given by Kelvin (Lamb, ${ }^{9}$ Sec. 162). This is the simplest mode of unsteadiness that can exist on a vortex ring. The rate of entrainment and detrainment of irrotational fluid into and out of the volume of fluid transported with the ring is presented as a function of ellipticity and core size. A qualitative comparison of the unstable manifold with flow visualization observations of turbulent vortex rings suggests that unsteadiness 
of the vortex core induces motions in the "atmosphere" of the ring that should not be interpreted as vortical. The ring of an elliptic cross section may also be useful in understanding aspects of fluid engulfment in the initial stages of pairing when two neighboring rings corotate and after the merging when an elongated core is formed.

The second case presented is that of two leapfrogging rings. In those experiments where the tracer is injected directly into the shear layer at the lip, there is little ambiguity between the vorticity and the tracer, aside from Schmidt number effects. Indeed, for such cases, core shapes obtained with contour dynamics calculations ${ }^{10}$ correspond well with flow visualization experiments. But what happens if a tracer is injected not only into the shear layer emitted from the orifice but also into the nonvortical fluid outside the cores? The vorticity may induce tracer particle motions in the irrotational region that can be confused as being associated with the vorticity. We will show this to be case in the experiment by Yamada and Matsui. ${ }^{11}$ It will be suggested that the descriptions that have been applied to their photographs ought to be reconsidered.

From a broader perspective, the term entrainment originates from work on laminar and turbulent shear flows, a jet, for instance, and refers to increasing mass flow along the main (horizontal, say) direction of flow. In the laminar case, momentum diffuses vertically, which leads to increasing horizontal mass flow; continuity then requires that this be fed by vertical flow into the layer. Turbulent entrainment, on the other hand, involves three steps: ${ }^{12}$ (i) the engulfment of irrotational fluid by the Biot-Savart induced velocity; (ii) vorticity mixing, i.e., the viscous diffusion of vorticity into the engulfed fluid; and (iii) the rejection of vorticity-mixed fluid into the previously irrotational part of the flow. Even in the absence of viscous diffusion [step (ii)], these processes imply growth of the vorticity containing region. The net result observed is the same as in the laminar case, namely, a streamwise increase of mass flux, although this is not apparent using the vorticity argument. The present work is restricted to entrainment and detrainment, in equal amounts, of irrotational fluid. Because the flows considered are time periodic, our work cannot say anything about permanent entrainment, however, it is hoped that recent generalizations of the manifolds to nonperiodic flows ${ }^{13-19}$ will do so in the near future.

The next section (Sec. II) defines the stable and unstable manifolds for time-periodic flows. Results are presented in Sec. III, and conclusions in Sec. IV.

\section{BACKGROUND}

In this section we aim to define the stable and unstable manifolds of dynamical systems theory. For a more detailed introduction, the reader is referred to the text by Guckenheimer and Holmes. ${ }^{20}$ Consider the equations governing the motion of particles in an $n$-dimensional unsteady flow:

$$
\dot{\mathbf{x}}=\mathbf{u}(\mathbf{x}, t), \quad \mathbf{x}, \mathbf{u} \in R^{n},
$$

with initial condition $\mathbf{x}(0)=\mathbf{x}_{0}$. For axisymmetric flow let $x$ and $r$ denote the axial and radial coordinate, respectively. In this case, the definition of the Stokes streamfunction $\psi$ :

$$
\dot{x}=\frac{1}{r} \frac{\partial \psi}{\partial r}, \quad \dot{r}=-\frac{1}{r} \frac{\partial \psi}{\partial x},
$$

together with the transformation $\eta=r^{2} / 2$, can be used to write (1) in Hamiltonian form:

$$
\dot{x}=\frac{\partial \psi}{\partial \eta}, \quad \dot{\eta}=-\frac{\partial \psi}{\partial x} .
$$

This fact opens up the use of various techniques developed for dynamical systems with a time dependent Hamiltonian. ${ }^{20}$ The solution to (1), called a pathline, is a curve in $\mathbf{x}$ space (the phase space) parametrized by time. The curves for different initial conditions $\mathbf{x}_{0}$ may intersect (albeit only at different $t$ ), and this impairs geometric thinking. To overcome this difficulty, time is introduced as an extra direction in the phase space. For this purpose (1) is written as an autonomous system (with the right hand side not explicitly dependent on time) at the expense of increasing the dimension of the phase space by one:

$$
\dot{\mathbf{x}}=\mathbf{u}(\mathbf{x}, \widetilde{\phi}), \quad \dot{\widetilde{\phi}}=1 .
$$

The solution is represented by curves in $(\mathbf{x}, \widetilde{\phi})$ space that do not intersect. If the velocity field is time periodic with period $2 \pi$ say, then to guarantee that trajectories do not intersect, it is sufficient to take $\phi=\widetilde{\phi} \bmod 2 \pi$ to be the extra dimension in phase space. To see this, assume that two different trajectories coincide at $\mathbf{x}_{1}, \phi_{1} \in[0,2 \pi]$. Then if the velocity field is time periodic, the two trajectories will continue to coincide for both time running forward and backward.

Discrete maps are an important class of dynamical systems. They may be studied in their own right or they may arise from a continuous dynamical system. For example, the cases to be presented have periodic unsteadiness and we shall be concerned with how particles are mapped in successive periods. After integrating (4) either numerically or exactly, from phase $\phi_{0}$ at period $i$ to the same phase at period $i+1$, we obtain

$$
\mathbf{x}^{i+1}=\mathbf{g}\left(\mathbf{x}^{i}\right), \quad \mathbf{x} \in R^{n} .
$$

Such a map is called a Poincaré map and $\phi_{0}$ will be called the base phase of the map.

We now wish to define the fixed points and manifolds of the Poincaré map, $\mathbf{g}(\mathbf{x})$. To save space we will simultaneously consider fixed points and manifolds of the steady flow,

$$
\dot{\mathbf{x}}=\mathbf{f}(\mathbf{x}), \quad \mathbf{x} \in R^{n} .
$$

The steady flow and map should not be thought of as being related to each other. Fixed points $\overline{\mathbf{x}}$ of (6) and (5) are defined, respectively, by

$$
\begin{aligned}
& \mathbf{f}(\overline{\mathbf{x}})=0 ; \\
& \mathbf{g}(\overline{\mathbf{x}})-\overline{\mathbf{x}}=0 .
\end{aligned}
$$

Note that the autonomous system (4) has no fixed points (since $\dot{\tilde{\phi}}$ never vanishes). However, it can have periodic solutions, i.e., particles whose trajectory follows a closed 
curve. Fixed points of the Poincaré map correspond to such solutions. The systems linearized about the fixed points are

$$
\begin{aligned}
& \dot{\xi}=\mathrm{A} \xi ; \\
& \xi^{i+1}=\mathrm{B} \xi^{i},
\end{aligned}
$$

where $\xi \equiv \mathbf{x}-\overline{\mathbf{x}}$ and $\mathrm{A}$ and $\mathrm{B}$ are constant $n \times n$ matrices with elements

$$
\begin{aligned}
& a_{j k}=\left.\frac{\partial f_{j}}{\partial x_{k}}\right|_{\mathbf{x}=\overline{\mathbf{x}}} ; \\
& b_{j k}=\left.\frac{\partial g_{j}}{\partial x_{k}}\right|_{\mathbf{x}=\overline{\mathbf{x}}} .
\end{aligned}
$$

The linearized systems lead to the notion of eigenspaces. For the continuous system, divide the generalized eigenvectors ${ }^{38}$ of A into three groups, having eigenvalues with real parts positive, negative, and zero, respectively. Each group spans a subset of $R^{n}$, referred to as the stable, unstable, and center eigenspaces, denoted as $E^{s}, E^{u}$, and $E^{c}$, respectively. For maps, these eigenspaces are constructed from the generalized eigenvectors of $B$ associated with eigenvalues having modulus $>1,<1$, and $=1$, respectively. A fixed point having no eigenvalue with a zero real part in the case of a continuous system or having no eigenvalue with unit modulus in the case of a map is called hyperbolic. Hyperbolic fixed points are nice because, not only is the existence of the stable and unstable manifolds guaranteed, but the Hartman-Grobman theorem (Ref. 20, p. 13) says that near a hyperbolic fixed point, the solution of the linearized and full system are qualitatively similar. In particular, they can be deformed to each other in a continuous, invertible, and one-to-one fashion. An important case of nonhyperbolic fixed points is the surface of a rigid no-slip surface. The conditions for manifold emanation in this case must be obtained separately. ${ }^{21}$

An important property of the eigenspaces is that each is an invariant subspace for the linearized system; that is to say a solution on each set remains there always for $-\infty<t<\infty$ for the time continuous systems and $-\infty<i<\infty$ for maps. This is so because the general solution of (6) and (5) is a linear combination of the generalized eigenvectors.

For hyperbolic fixed points the stable and unstable manifolds of $\overline{\mathbf{x}}$, denoted as $W^{s}(\overline{\mathbf{x}})$ and $W^{u}(\overline{\mathbf{x}})$, respectively, are simply nonlinear extensions of the eigenspaces. One begins by defining the manifolds locally in some region $\mathcal{U}$ (not necessarily small) containing $\overline{\mathbf{x}}: W_{\text {loc }}^{\text {s }}(\overline{\mathbf{x}})$ is the set of all solutions in $\mathcal{U}$ that tend to $\overline{\mathbf{x}}$ as $t \rightarrow \infty$ and never leave $\mathcal{U}$ for $t \geqslant 0$. Similarly, $W_{\text {loc }}^{u}(\overline{\mathbf{x}})$ is the set of all orbits in $\mathcal{U}$ that tend to $\overline{\mathbf{x}}$ as $t \rightarrow-\infty$ and never leave $\mathcal{U}$ for $t \leqslant 0$. This definition is general and does not refer to the eigenspaces, but the stable manifold theorem for continuous systems as well as maps asserts that for a hyperbolic fixed point $W_{\mathrm{loc}}^{s}(\overline{\mathbf{x}})$ and $W_{\mathrm{loc}}^{u}(\overline{\mathbf{x}})$ exist, are tangent to $E^{s}(\overline{\mathbf{x}})$ and $E^{u}(\overline{\mathbf{x}})$ at $\overline{\mathbf{x}}$, and have the same dimensions $n_{s}$ and $n_{u}$. In either case, the global $W^{u}(\bar{x})$ is then defined constructively by letting the local unstable manifold flow forward under the dynamical system. Similarly, the global stable manifold is constructed by letting the corresponding local manifold flow backward in time.

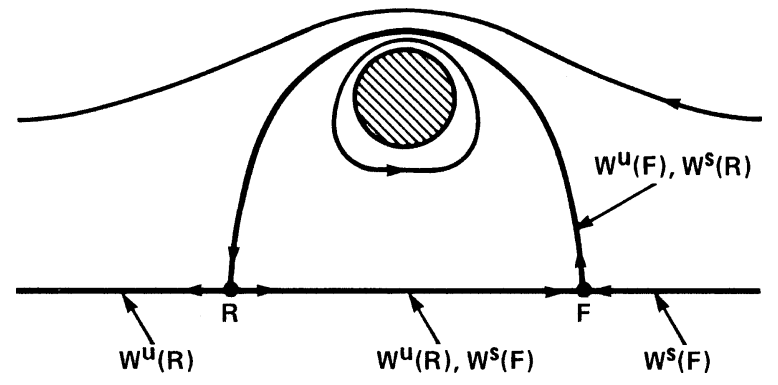

FIG. 1. Sketch of streamlines for steadily translating vortex rings. The hatched region shows the vorticity containing region.

We noted earlier that a fixed point of the Poincaré map corresponds to a periodic solution of the autonomous system (4). The fact that a hyperbolic fixed point of the Poincaré map has stable and unstable manifolds means that a hyperbolic periodic solution of the autonomous system also has stable and unstable manifolds. These are surfaces of dimension $n$ that exist in the space $\left(\mathbf{x}, \phi_{0}\right)$. They are formed by letting the base phase $\phi_{0}$ of the Poincaré map take on all possible values in a period.

By construction, the manifolds are invariant subspaces; they always contain the same fluid particles. However, they are not the only invariant subspaces. Consider a streakline, defined as the locus of tracer particles injected at the same location, $\mathbf{x}_{1}$ say. If we slightly generalize this definition by allowing the particles to flow both backward and forward in time, then a streakline is also an invariant subspace. In a time-periodic flow, invariant surfaces necessarily undergo time-periodic oscillation.

To illustrate these definitions, consider the steady flow vortex ring in a reference frame traveling with the ring (Fig. 1). The dividing streamline is both the unstable manifold of $F$ (excluding the point $R$ ) and the stable manifold of $R$ (excluding the point $F$ ). Connections such as $\overline{R F}$ and the dividing streamline that join two saddles are called heteroclinic orbits and together they form a heteroclinic cycle. In the present case, the unstable and stable manifolds of the forward and rear stagnation points coincide. This situation is highly exceptional; if the ring were slightly disturbed, the body of fluid carried with it would leak. In the time-periodic case, this will manifest itself as a splitting of the manifolds of the Poincaré map. In the early 1970s several papers motivated their study of vortex rings by suggesting that vortex rings could be used to transport chimney wastes to high altitudes. We should be thankful that the scheme was never implemented.

\section{RESULTS}

\section{A. Elliptical core ring: Flow definition}

The first example is that of a single unsteady vortex ring of circulation $\Gamma$. We consider the solution due of Moore ${ }^{8}$ in which the core is an ellipse of uniform vorticity with semimajor and semiminor axes, $a$ and $b$. The solution is valid in the limit of thin cores (compared to the ring radius). The flow is therefore two dimensional locally and the core rotates at 
the constant angular velocity of Kirchhoff's two-dimensional elliptic vortex (Lamb, ${ }^{9}$ Sec. 159). The angle $\theta$ of the major axis of the ellipse is given by

$$
\theta(t)=\frac{\Gamma t}{\pi(a+b)^{2}} \text {. }
$$

This rotation of the core induces oscillations in the speed $U(t)$ of translation of the ring:

$$
U(t)=\frac{\Gamma}{4 \pi R}\left[\log \left(\frac{16 R}{a+b}\right)-\frac{1}{4}+\frac{3}{2} \frac{(a-b)}{(a+b)} \cos 2 \theta\right] .
$$

Here $R$ is the "impulse radius" of the ring defined in terms of the conserved impulse, $P$ by $R=\sqrt{P / \pi \Gamma}$. The impulse radius is a constant, however, the radial centroid $r_{c}$ of the core undergoes oscillations given by

$$
\frac{d r_{\mathrm{c}}}{d t}=\frac{\Gamma}{4 \pi R}\left(-\frac{3}{2} \frac{(a-b)}{(a+b)} \sin 2 \theta\right) .
$$

In the figures, the normalizing length $r_{0}$ denotes the value of the radial centroid at $t=0$ when the major axis of the ellipse is parallel to the symmetry axis. Note that the period of oscillations corresponds to a half rotation of the major axis. In Ref. 10, deviations from Moore's solution were investigated for thick cores, and it was found that strain due to curvature caused the aspect ratio of the ellipse to pulsate as well as to decrease secularly. Thus, for fat cores the results of this paper apply for only a few periods.

In order to track particles, one needs the velocity field. However, in Moore's study it was necessary to know only the zeroth-order streamfunction in the vicinity of the core, and the corresponding velocities have $\mathcal{O}(1)$ errors. In the present calculations we obtain the velocities everywhere numerically from the contour dynamics equations. ${ }^{10}$ Thus, there is the slight inconsistency that the core dynamics follows an asymptotic result whereas the velocity field is unapproximated.

The Poincaré map is defined using the period of the velocity field, which is half the period of rotation of the major axis and the base phase is taken to be when the major axis is parallel to the symmetry axis (i.e., when $\theta=0$ ). The unstable manifold is computed by first obtaining, using secant iterations, the location $x_{F}$ of the forward hyperbolic fixed of the Poincaré map lying on the symmetry axis. Next, a particle lying at $\left(x_{F}, \epsilon\right)$, i.e., a small distance radially away from the fixed point, is mapped forward one period. This defines two endpoints of a segment. Many points are placed on the segment and iterated forward to build the manifold. The fact that the initial segment does not lie exactly on the manifold poses no practical problems. Since neighboring points converge to the manifold from both sides, initial errors are quickly diminished. This was checked by deliberately introducing large errors into the initial angle of the segment and comparing with the shape of the manifold without the errors. The stable manifold of the rear fixed point is obtained by symmetry. If time were reversed, the vortex would rotate in a clockwise direction and $R$ would be the front hyperbolic point and a

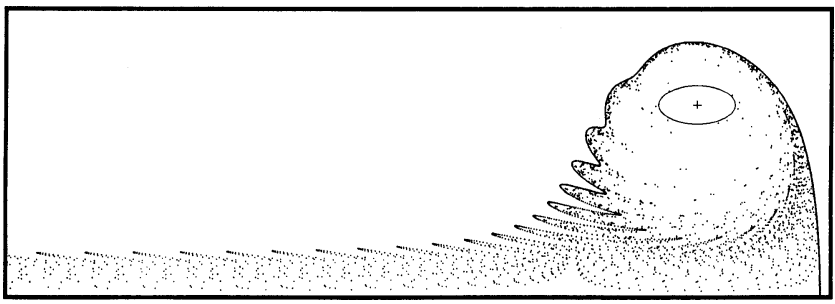

FIG. 2. Unstable manifold of the forward periodic point for an elliptical core ring. $\lambda=2, \alpha \equiv(a+b) /\left(2 r_{0}\right)=0.15$.

similar procedure would yield its unstable manifold symmetric to the first one. With time restored to its original course, this becomes the stable manifold of $R$.

\section{B. Elliptical core ring: Analysis of manifolds}

Figure 2 shows the unstable manifold for $\alpha \equiv(a$ $+b) /\left(2 r_{0}\right)=0.15$ and an aspect ratio $\lambda \equiv a / b=2$. Figure 3 shows a limited portion of both manifolds. The unstable manifold of $F$ begins as nonoscillatory but meanders about the stable manifold of $R$ in the rear of the ring. The stable manifold of $R$ begins as oscillatory in the front of the ring and becomes nonoscillatory in the rear. The square symbols mark some intersection points. Because the manifolds intersect the Smale-Birkhoff test implies that the horseshoe type of chaos is present. $^{20}$

A particle started on one manifold ends up somewhere else on the same manifold after one period. Hence, intersection points get mapped to intersection points. It was checked that the square symbol nearest $F$ is mapped to the third, the second to the fourth, etc. Therefore the lobe denoted as $A_{0}$ is mapped to $A_{1}$, and so on. All the lobes must have the same volume, and this was verified. After the sixth period, fluid in $A_{0}$ is engulfed (as $A_{6}$ ) into the oval shaped region $O$, consisting of the nonoscillating halves of the two manifolds. For convenience we shall refer to a lobe such as $A_{6}$ as the entrance lobe. Similarly, $B_{0}$ is "detrained" (as $\left.B_{5}\right)$ after the fifth

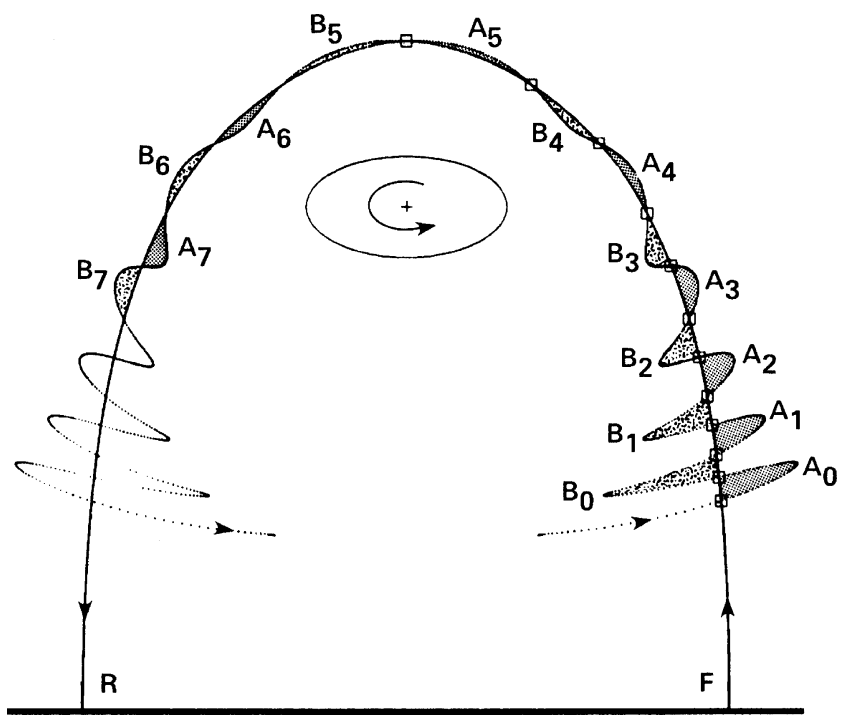

FIG. 3. Abridged portions of the stable and unstable manifolds corresponding to the previous figure illustrating fluid engulfment and rejection. 


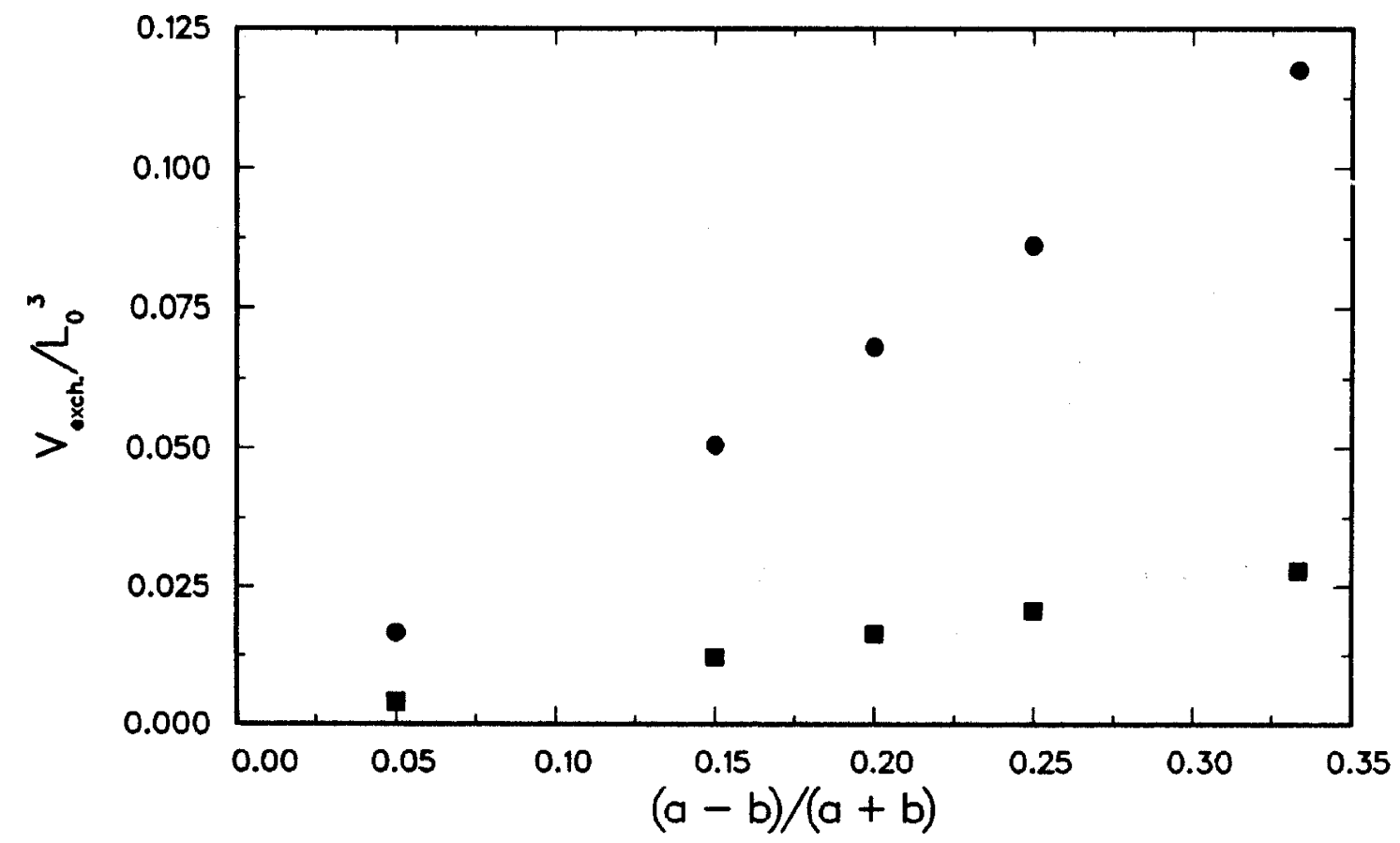

FIG. 4. Volume of fluid exchanged by elliptical core vortex rings per diameter translation as a function of the ellipticity. $\bullet, \alpha \equiv(a+b) /\left(2 r_{0}\right)=0.15 ; \mathbf{\square}, \alpha$ $=0.125$.

period. A lobe such as $B_{5}$ shall be referred to as the exit lobe. Thus, in every period the region $O$ picks up fresh fluid in exchange for old fluid over a limited portion of its boundary.

Figure 4 plots the volume exchanged as a function of the oscillation amplitude $\epsilon_{e} \equiv(a-b) /(a+b)$ during the time that the vortex travels one diameter. The largest value of $\epsilon_{e}$ shown corresponds to an aspect ratio of two. The solid circles are for a core size to radius ratio $\alpha=0.15$ and the solid squares are for $\alpha=0.125$. According to the linearized theory of Melnikov, ${ }^{20}$ valid for small perturbations about the steady state, the amplitude of the manifold oscillations is linear in the perturbation amplitude $\epsilon_{e}$. In the present cases, the linear behavior persists up to quite large values of the ellipticity. Note that the time that it takes to travel one diameter is independent of $\epsilon_{e}$; hence the ordinate scaling does not affect the linearity. Also note the sensitivity of the slope to $\alpha$ : a slightly thicker core processes fluid much more rapidly.

More intersections of the two manifolds give information about smaller volumes of fluid. Figure 5 sketches repre- sentative manifolds with as many lobes shown as the fineness of a pen would allow. The sketches assume a particular topology: the entrance lobe intersects its symmetric image after $n_{\text {int }}=3$ periods. In the computations, $n_{\text {int }}$ is larger than this, $\mathcal{O}(10)$ in Fig. 2, for instance. Suppose we wish to know how long particles remain with the vortex after they are engulfed. Rom Kedar et al. ${ }^{5}$ have shown that this information is completely contained in subregions of the entrance lobe. By observing successive maps of the entrance lobe, one sees that it flows counterclockwise around the vortex and every map after the third has a piece contained in the exit lobe that has been rejected. Consider the shaded subregions of the entrance lobe. Those regions, like the stippled and screendotted, that happen to be contained in a single lobe of the stable manifold, have relatively simple histories. The stippled region is mapped out of $O$ after four periods, the screen-dotted after five. Following their motion backward in time, one observes that their shapes are symmetric. Note, however, that their orientation changes. The solid region is

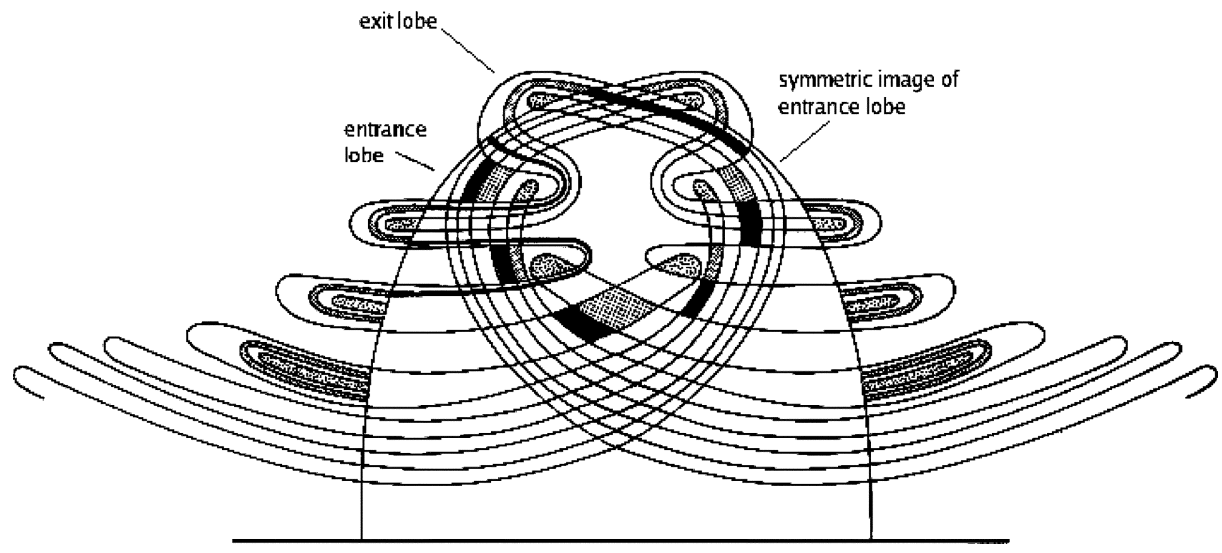

FIG. 5. Hand sketch illustrating the motion of subsets of the entrance lobe. 

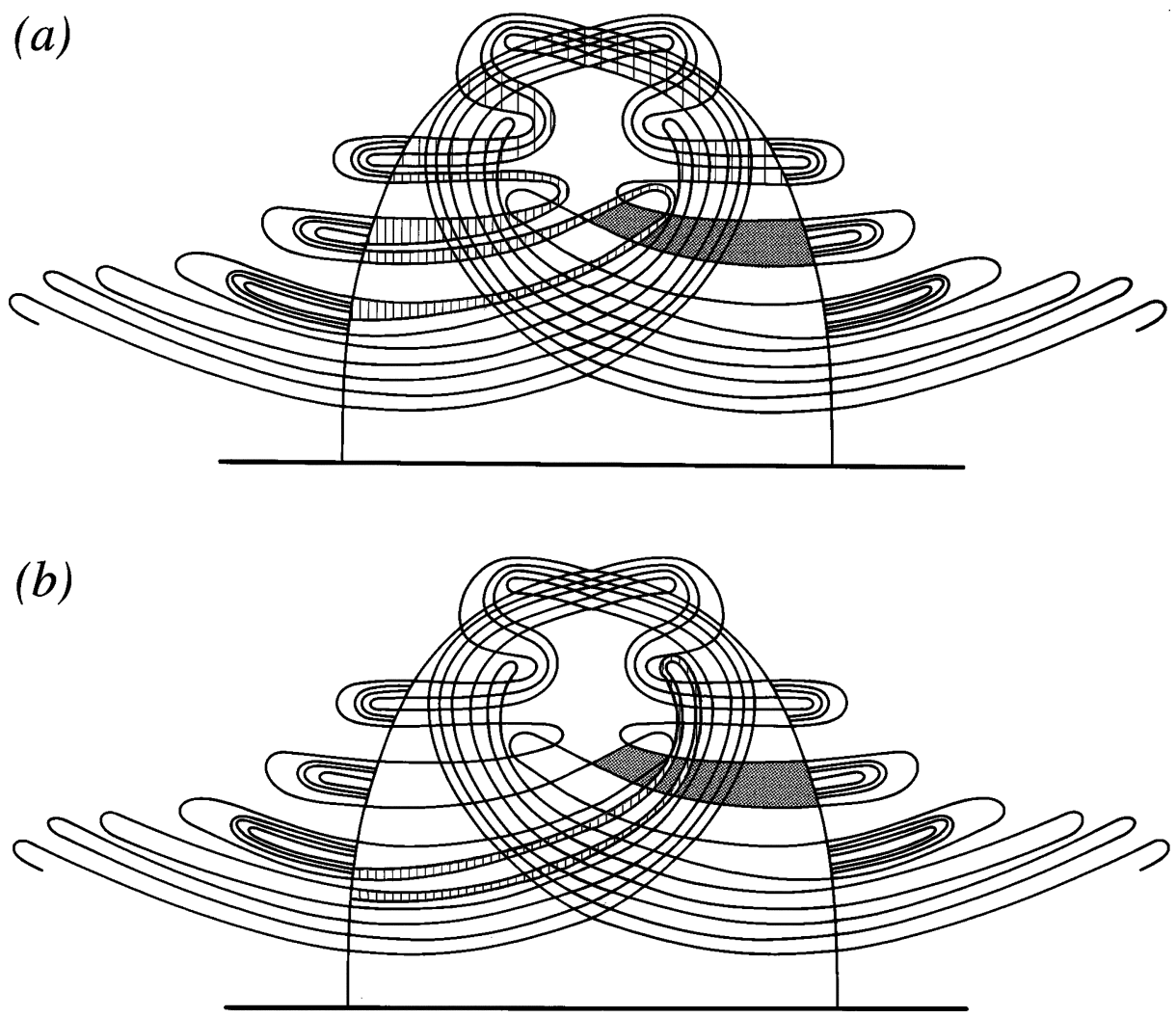

FIG. 6. Hand sketch of six Poincaré maps of a "rectangle" (screen dotted) leading to a the horseshoe map. The iterates are hatched and proceed counterclockwise. (a) shows the first five iterations and (b) the last. not inside one lobe of the stable manifold, but rather very many that are not drawn. It has a more complicated history. Following its motion forward, it intersects a stable manifold lobe that is drawn after four iterations. We identify its middle piece as the first to be rejected in six more iterations. Following it further in time as it intersects more of the drawn stable manifold lobes, one is able to identify more portions to be detrained in later iterations. Note how the solid region is drawn out along the unstable manifold and begins to reveal its shape.

Figures 6(a) and 6(b) gives an example of how the horseshoe arises. It depicts iterations (hatched) of a topologically rectangular region (screen-dotted) whose boundaries lie in the stable and unstable manifolds. Note how the region is bent and after the sixth iteration [Fig. 6(b)] intersects itself in two strips. In six more iterations, this fluid will intersect the original rectangle in four finer strips contained in the original two, and so on. Rectangles that have this property are said to form a Markov partition for the Poincaré map. Note again how the rectangle is drawn out along the unstable manifold, and more iterations would reveal its finer structure.

\section{Implications for interpreting flow visualizations of turbulent rings}

Figure 7 is a spark-schlieren photograph kindly provided to us by Sturtevant [the same as Fig. 11(d) in Ref. 22]. It shows a shock-tube generated ring propagating to the left. In a schlieren image, the difference in illumination at a given point from the overall illumination is proportional to the density gradient, normal to a knife edge, integrated over the entire length of the test section normal to the photograph.
Unfortunately, the direction of the knife edge is not provided. The walls of the tube were cooled to aid in visualization. The vortical core consists of cooled shear layer fluid as well as warm ambient fluid sandwiched between turns of the spiral.

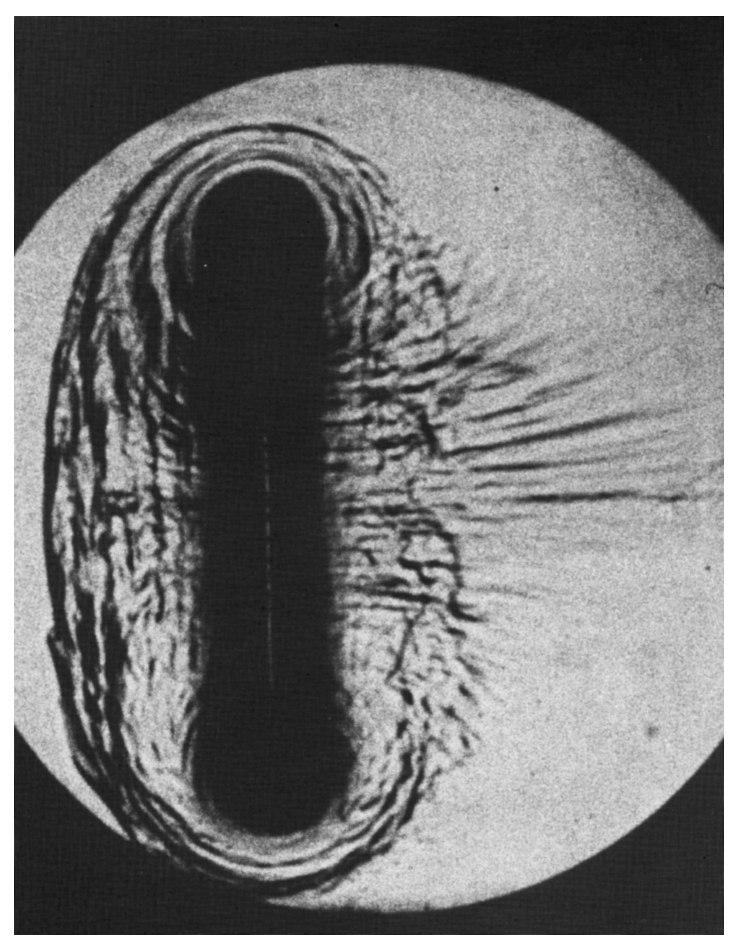

FIG. 7. Schlieren visualization of a shock tube generated ring propagating to the left. Courtesy of B. Sturtevant. 
As temperature mixes at roughly the same rate as vorticity, the vortex subcore ${ }^{23}$ acquires a smooth temperature distribution. The visualization is also aided by the reduced density in the vortical core from compressibility effects. In any case, the vortical core is the dark region and outer undiffused turns of the spiral also appear to be visible.

We are interested in the streaky pattern in the rear that is described by Sturtevant in a later report. ${ }^{24}$ This description applies to oblique views of the ring that are of insufficient resolution to allow reproduction here, however, they refer to a realization in which the same generation parameters and visualization technique were used as for the photograph we have presented. We think it is not inappropriate to quote that description in its entirety. It should be noted that Sturtevant uses "ring" to refer to the entire volume of fluid, vortical and nonvortical (or weakly vortical) carried with the ring.

"In the second photograph $(x / D=3.1)$ (the photograph we have been able to provide is slightly earlier at $x / D$ $=2.78$ ) the circumferential lines on the external interface have distorted and have developed a three-dimensional irregularity. The ring now trails a thin wake.

"At $x / D=4.5$ the flow inside the ring seems to be fully turbulent. Disturbances on the boundary of the ring protrude into the surrounding fluid and, after being convected along the boundary to the rear of the ring, seem to grow almost explosively outward from the rearward surface of the ring. Apparently, this is a mechanism for the ejection of fluid into the wake of the vortex ring, because in this photograph, and in subsequent ones, the wake thickens very rapidly. The rapid growth of disturbances on the interface at the rear of the vortex ring appears to be the mechanism, not only for ejecting ring fluid into the wake, but also for entraining external fluid into the ring, because, after $x / D=6.0$ mixing within the ring becomes so strong that the photographs rapidly lose their contrast. By $x / D=6.0$, the spatial distribution of the inhomogeneities within the ring seems to have become relatively homogeneous and isotropic. It is noteworthy that this state of fully developed turbulence is reached just before the instability waves on the core of the ring reach a substantial amplitude. Though the relationship between the core instability and the turbulence in the ring is not at all clear, it is certainly apparent from these photographs that substantial three-dimensional random, unsteady motion (turbulence) occurs within the ring before there are any signs of the fundamental core instability" (Sturtevant's italics).

It should be noted that Fig. 12 in Ref. 22 is a photograph of the same case without precooling. Only the naturally occurring reduced density in the vortex core is used for visualization. Significantly, no streaks are present, suggesting that they are either irrotational or weakly vortical.

Surtevant's description of the interface is consistent with the picture of entrainment and detrainment provided by the unstable manifolds. Wherever turbulence is mentioned, it does not refer to vortical motions but rather chaotic motion of the cold fluid carried with the ring and entrained ambient warm fluid. The distortions of the interface are described as three-dimensional, suggesting that the unsteady vortical motion causing it is also three dimensional. This seems inconsistent with the fact that it is observed before the core $\left(\right.$ Widnall $^{25}$ ) instability. Several possibilities may account for this. There may be another three-dimensional mode on the core that is inducing these motions. A second possibility is that since schlieren visualization presents an integrated view of density gradient, complex axisymmetric stirring of cold and ambient fluid is being mistaken for three-dimensional motion. Even when it does appear, the Widnall instability is an inefficient stirrer because the instability wave is stagnant in the linear phase, according to both experiment ${ }^{26}$ and theory. ${ }^{25}$ Even when the waves begin to rotate ${ }^{26}$ in the nonlinear phase, short waves have a short range effect on the velocity field. This is suggested by Widnall, Bliss, and Tsai, ${ }^{25}$ who say that "for short waves, such as are observed on vortex rings, the velocities induced at the core boundary owing to distant perturbations on the ring are negligible; preliminary calculations of the outer potential flow using toroidal coordinates indicate that these are of order $1 /\left(N_{w} R\right)^{2}$ as $N_{w} R \rightarrow \infty$." Here $N_{w}$ is the number of waves and $R$ is the radius of the ring.

What form of unsteadiness produces the stirring in the experiments is still an open question. One possibility is residual unsteadiness from the formation of the ring. Another possibility is the instability of two-dimensional compressible vortices studied by Broadbent and Moore. ${ }^{27}$ They found that a two-dimensional circular patch of uniform vorticity and entropy was unstable to two-dimensional wavy deformations of the boundary, the elliptic mode being the most unstable. The instability is weak; it takes many core rotations for the initial disturbance to undergo an $e$-fold amplification especially at low Mach numbers based on maximum rotational velocity in the core. Sturtevant mentions that the maximum velocity in the core is near sonic; this allows us to find the growth rate of the elliptic mode from Table 1 of Ref. 27. Then, using Kelvin's speed formula with a core size to radius ratio of 0.10 estimated by Sturtevant, we find that in the time that the vortex propagates one ring diameter there are $0.90 e$ foldings of the initial disturbance. On the other hand, using Eq. (9.2) of Ref. 28, the three-dimensional instability undergoes $1.4 e$ foldings, but, as mentioned earlier, its influence decays more rapidly from the core and it does not rotate. Thus, one cannot a priori discount the presence and influence of the Broadbent and Moore instability.

Maxworthy (Ref. 29, hereafter M) studied turbulent vortex rings in water with $\mathrm{Re} \approx 1 \times 10^{4}$ based on propagation velocity and toroidal radius. Figure M:3 shows a ring marked with a blob of dye. The blob becomes puffy and its boundary corrugated with ejections similar to those suggested by the unstable manifold. Most of the dye is rejected to a wake and eventually remains only in a thin toriodal core.

In Fig. M:4 an undyed ring is pushed through a patch of dye. "The outer region of the moving bubble was immediately filled with dyed fluid, but the core remained clear. As time progressed, a thin skin around the core became dyed but penetration to the center of the core never seemed to take place, at least during our experiments." The remarks suggest that there is little exchange between the vortical core and the surrounding fluid.

In Fig. M:5 a weak salt solution was used to mark the bubble and observed using the shadowgraph technique. "The 


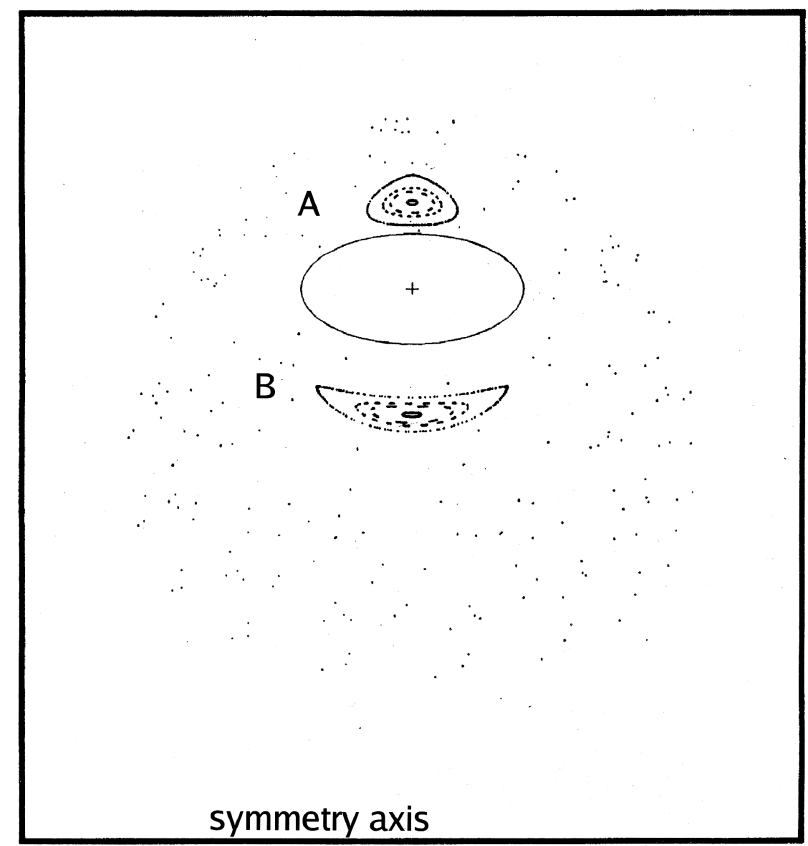

FIG. 8. Poincaré section of regions of fluid permanently carried by an elliptical core ring. The region marked " $\mathrm{A}$ " is transported to " $\mathrm{B}$ " and " $\mathrm{B}$ " is transported to "A" after half a core rotation. $\lambda=2, \alpha \equiv(a+b) /\left(2 r_{0}\right)=0.15$.

major motions in the outer bubble are of a larger scale; they mix environmental with bubble fluid and deposit the majority into a wake. There are, clearly, small scale streaks in the region, but are being convected around and stretched by the large scales, and only show up because of the small diffusion coefficient possessed by the denser salty water."

These observations are also consistent with unsteady motion of a vortical core inducing the entrainment and detrainment.

Unlike the situation we have treated, in the experiments of Maxworthy, the vortical core is not completely isolated from the surrounding fluid. We have not addressed slow permanent entrainment characterized by growth of the bubble. The rate of growth of bubble volume divided by the surface area times the propagation velocity defines an entrainment coefficient. Maxworthy reports a value of about 0.01 , independent of $R e$. Weak vorticity either diffusing into the bubble or entering it via wisps torn off from the core would become turbulent due to chaotic passive advection. This vorticity is continually being rejected into the wake, resulting in a slow loss of impulse.

\section{Fluid carried permanently with the ring}

Despite the fluid exchange process, some fluid is permanently carried with the vortex. Figure 8 shows that two such regions exist near the core. After half a major axis rotation region " $\mathrm{A}$ " is transported to " $\mathrm{B}$," and vice versa. The motion is quasiperiodic and periodic for some exceptional points inside. In a cylindrical coordinate phase space in which the azimuthal direction is chosen to be angle of the ellipse, the motion of particles takes place on tori whose cross section has been depicted in the figure. These are called KAM tori after the Kolmogorov-Arnold-Moser theorem for perturbed
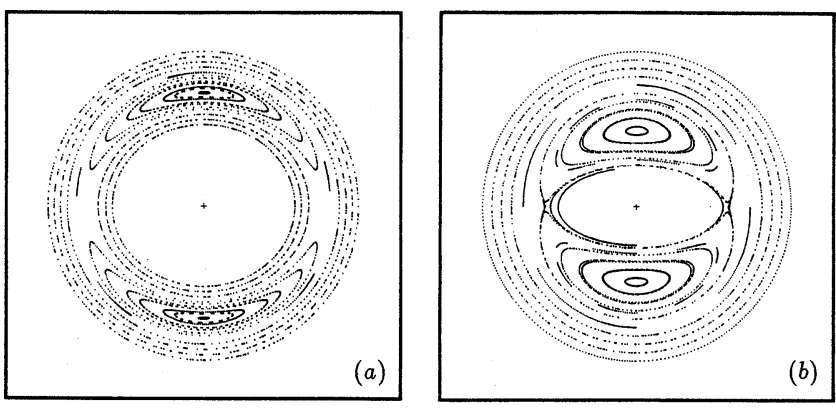

FIG. 9. Poincaré section of particle paths for Kirchhoff's two-dimensional (planar) elliptic vortex in a reference frame rotating with the vortex. The boundary of the vortex core is not shown but corresponds roughly to the innermost ellipse. (a) $\lambda=1.1$; (b) $\lambda=2$.

Hamiltonian systems. ${ }^{20}$ In the fluid mechanical context, the KAM theorem refers to the survival, under small perturbations about steady flow, of such regions near the closed streamlines of the steady flow.

The existence of these regions of trapped fluid near the vortex core can be understood in terms of the streamline pattern of the steadily rotating Kirchhoff elliptic vortex in two dimensions. This is because the velocity field in the vicinity of the core is locally the same with additional terms in the axisymmetric case that account for self-induction. From the KAM theorem one expects that some of the qualitative features will remain unchanged in the presence of these additional terms. Figure 9(a) shows the Poincaré section, every half rotation, of particle paths relative to the vortex for a slightly elliptical vortex. We used the velocity field of the Kirchhoff elliptic vortex as neatly written down by Saffman ${ }^{30}$ using complex variables. If the vortex were circular, particle paths would also be circular, but the slight ellipticity creates mounds of fluid on the major axis side that rotate with the vortex. Similar mounds exist for the potential flow of a solid rotating ellipse. ${ }^{31}$ The mound is created about the point in the circular flow where the particle rotation frequency is the same as the vortex rotation frequency. Figure 9(b) shows larger mounds for an aspect ratio equal to two. These mounds erode in the presence of waves Love ${ }^{32}$ waves on the boundary of the elliptic vortex. ${ }^{10}$

\section{E. Leapfrogging rings}

Finally, the leapfrogging of two vortex rings is considered. For the vorticity dynamics, the simple model of Dyson $^{33}$ is used. In this model, core deformation is neglected, and the self-induced velocity of each ring is given by Kelvin's formula (Lamb, ${ }^{9}$ Sec. 163) while the mutually induced velocity is obtained by assuming zero thickness cores. This is a valid assumption when the vortex rings cores are separated by a distance larger than their core radii. Classical works provide only the streamfunction for a zero thickness ring, however, the induced velocity can be easily obtained in closed form (see Ref. 10, p. 87) by using the BiotSavart law.

The case considered has a core size to radius ratio of 0.1 and an initial separation of one radius. Axisymmetric contour dynamics, which allows core deformation, shows that Dys- 

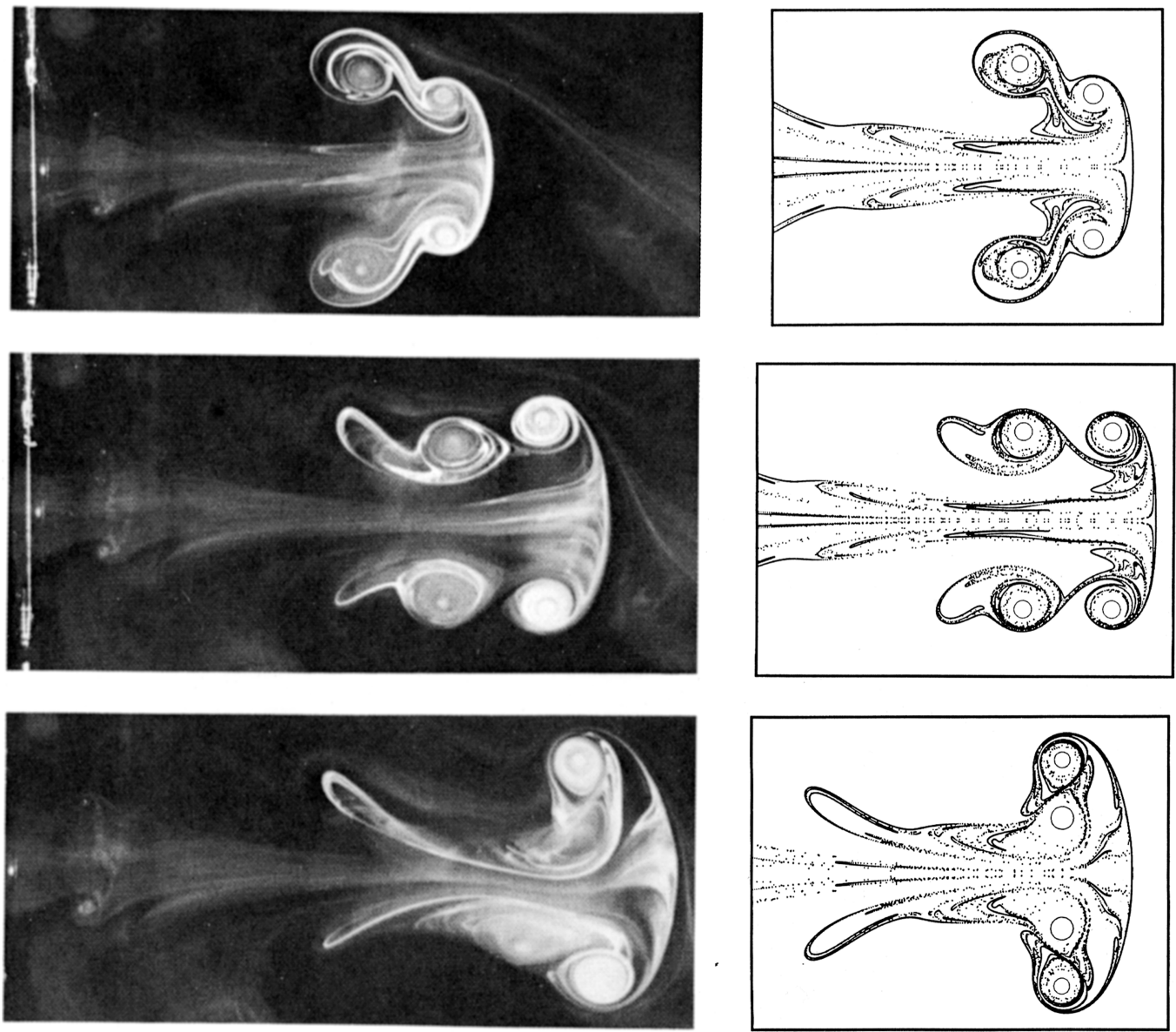

FIG. 10. Unstable manifold at different phases for two leapfrogging rings. Photographs are from Yamada and Matsui (Ref. 11).

on's model gives an excellent result for this case. ${ }^{10}$ Figure 10 shows the unstable manifolds of the Poincaré map with period equal to one passage at three phases; the circles indicate the vortical core. The flow visualization photographs are from Yamada and Matsui. ${ }^{11}$ The stretching along the manifold is so rapid that even though 4000 particles were placed on the initial segment, the visual appearance of the manifold as a connected curve disappears after the third forward map of the initial segment. For the middle photograph, the manifold winds back and forth between the two vortices, each time passing through the "braid" region.

Even the very fine scale features of the manifold agree remarkably well with the experimental photographs. Maxworthy ${ }^{34}$ used the photographs together with vorticity diffusion arguments to provide a plausible rendering of the underlying vorticity. The first photograph shows the first passage almost completed, and according to him "thereafter, the latter (the passing vortex) is distorted and wraps around the former, and the two rings become one." In referring to the third photograph, Maxworthy says that the passing vortex "has become so distorted that it is barely recognizable," and Yamada and Matsui say that "the core of the first ring was severely deformed and stretched, and it seemed to roll up around that of the second ring...." Maxworthy's guess of the vorticity field underlying the third photograph is sketched as Stage 3 in Fig. 1 of his paper and shows the two rings diffused together.
On the other hand, the present result suggests that the observed pattern may be due merely to complex motion of tracer in irrotational or weakly vortical fluid, with vortex cores behaving in a simple, nondeforming and almost classical manner. It is only the tracer that appears to deform and roll up around the leading vortex.

This suggestion should be tempered with three points. First, our type of interpretation is appropriate only for this experiment in which a smoke wire was stretched across the entire diameter of the orifice, causing smoke to be introduced, not only in the emitted shear layer (which would mark the vorticity aside from Schmidt number effects) but also into nonvortical fluid initially transported with each vortex. If one is careful to ensure that only vortical regions are marked then there is less danger in identifying smoke with vorticity. Second, weak vorticity around the periphery of a core may also become susceptible to passive advection and acquire the structure of the unstable manifold. Third, the question of whether at the Reynolds number of the experiment (1600 based on initial translation speed and orifice diameter) the two rings diffuse together or behave in the simple manner suggested at the Reynolds number of the experiment will have to await viscous numerical computations at higher Reynolds number. Stanaway et al. ${ }^{35}$ simulated a passage interaction for the same core size (based on where the maximum velocity occurs) and vortex separation as the present case. The Reynolds number based on initial self- 
induced speed and diameter was 609 . They observed that the first passage is successful but, during the second, the passing vortex strongly deforms. A measure of the extent of viscous merging is the level of the highest vorticity contour that surrounds the vorticity peaks of both rings. At roughly the phase of the last Yamada and Matsui photograph and stage 3, as sketched in Maxworthy, ${ }^{35}$ this level is $10 \%$ of the peak vorticity. Hence, at the Reynolds number of the simulation, neither Maxworthy's nor the classical picture is completely accurate. We hope that simulations at a higher Reynolds number will lead to a synthesis of inviscid descriptions and Maxworthy's views about the role of viscous diffusion.

\section{CONCLUSION}

Several features present in observations of turbulent vortex rings, such as puffiness and striations in the tracer, a trailing wake, and the entrainment of fluid from a patch of dye placed in the path of an initially unmarked vortex ring, are revealed by the unstable manifold of an axisymmetric vortex ring with an elliptic core. This suggests that irrotational motions induced by simple forms of core unsteadiness may explain those features for turbulent rings. Qualitative agreement with experiment does not necessarily mean that a sufficient explanation has been offered. The boundary of the core may have an arbitrary number of axisymmetric waves and there may be azimuthal waves. Sallet and Widmayer ${ }^{36}$ have indeed measured irregular hot-wire signals for turbulent rings. We only wish to suggest that relatively simple unsteady models may be fruitful in elucidating large scale aspects of the mixing.

For two leapfrogging rings it is found that the unstable manifold reveals even fine scale patterns in the smoke suggests that blobs of fluid near the unstable manifold are drawn out along it and acquire its structure. The unstable manifold may therefore be useful as a tool for numerical flow visualization. The conventional approach to numerical flow visualization is to follow the trajectories of arbitrary clusters of particles. This has the disadvantage that it is expensive to place particles with sufficient resolution wherever dye is located initially. One usually starts with a judiciously selected blob, but this does not yield a global picture. The unstable manifold is a curve [or surface for three dimensional (3D) flow]: it is therefore easier to resolve and provides a more global picture. This conclusion is reinforced by the existence of analogs of the manifolds for general unsteady flows. These analogs are applied to vortex ring flows in the companion paper by Shadden, Dabiri, and Marsden. ${ }^{37}$

${ }^{1}$ H. Aref, "Stirring by chaotic advection," J. Fluid Mech. 143, 1 (1984).

${ }^{2}$ H. Aref and S. Balanchandar, "Chaotic advection in a Stokes flow," Phys. Fluids 29, 3515 (1986).

${ }^{3}$ J. Chaiken, R. Chevray, M. Tabor, and Q. Tan, "Experimental study of Lagrangian turbulence in a Stokes flow," Proc. R. Soc. London, Ser. A 408, 165 (1986)

${ }^{4}$ W.-L. Chien, H. Rising, and J. Ottino, "Laminar mixing and chaotic mixing in several cavity flows," J. Fluid Mech. 170, 355 (1986).
${ }^{5}$ V. Rom-Kedar, A. Leonard, and S. Wiggins, "An analytical study of transport, mixing, and chaos in an unsteady vortical flow," J. Fluid Mech. 214, 347 (1990).

${ }^{6} \mathrm{~T}$. Solomon, S. Tomas, and J. Warner, "Role of lobes in chaotic mixing of miscible and immiscible impurities," Phys. Rev. Lett. 77, 2682 (1996).

${ }^{7}$ D. Khakhar, H. Rising, and J. Ottino, "Analysis of chaotic mixing in two model systems," J. Fluid Mech. 172, 419 (1986).

${ }^{8}$ D. Moore, "The velocity of a vortex ring with a thin core of elliptical cross-section," Proc. R. Soc. London, Ser. A 370, 407 (1980).

${ }^{9}$ S. H. Lamb, Hydrodynamics (Dover, New York, 1932).

${ }^{10} \mathrm{~K}$. Shariff, A. Leonard, and J. Ferziger, "Dynamics of a class of vortex rings," TM 102257, NASA, 1989; also K. Shariff, Ph.D. thesis, Stanford University, 1989.

${ }^{11} \mathrm{H}$. Yamada and T. Matsui, "Preliminary study of mutual slip-through of a pair of vortices," Phys. Fluids 21, 292 (1978).

${ }^{12}$ P. Dimotakis, "Entrainment into a fully developed two-dimensional shear layer," 22nd Aerospace Sciences Meeting, AIAA Paper 84-0368, 1984.

${ }^{13}$ G. Haller and A. Poje, "Finite time transport in aperiodic flows," Physica D 119, 352 (1998).

${ }^{14} \mathrm{G}$. Haller, "Finding finite-time invariant manifolds in two-dimensional velocity fields," Chaos 10, 99 (2000).

${ }^{15} \mathrm{G}$. Haller and G. Yuan, "Lagrangian coherent structures and mixing in two-dimensional turbulence," Physica D 147, 352 (2000).

${ }^{16} \mathrm{G}$. Haller, "Distinguished material surfaces and coherent structures in three-dimensional fluid flows," Physica D 149, 248 (2001).

${ }^{17} \mathrm{C}$. Jones and S. Winkler, "Invariant manifolds and Lagrangian dynamics in the ocean and atmosphere," in Handbook of Dynamical Systems: Towards Applications, edited by B. Fiedler (Elsevier, Amsterdam, 2002), pp. 55-92.

${ }^{18}$ A. Mancho, S. Small, S. Wiggins, and K. Ide, "Computation of stable and unstable manifold of hyperbolic trajectories in two-dimensional aperiodically time-dependent vector fields," Physica D 182, 188 (2003).

${ }^{19} \mathrm{~S}$. Wiggins, "The dynamical systems approach to Lagrangian transport in oceanic flows," Annu. Rev. Fluid Mech. 37, 295 (2005).

${ }^{20}$ J. Guckenheimer and P. Holmes, Nonlinear Oscillations, Dynamical Systems, and Bifurcations of Vector Fields (Springer-Verlag, New York, 1983).

${ }^{21}$ K. Shariff, T. Pulliam, and J. Ottino, "A dynamical systems analysis of kinematics in the time periodic wake of a circular cylinder," in Vortex Dynamics and Vortex Methods, edited by C. Anderson and C. Greengard (American Mathematical Society, Providence, RI, 1991), Vol. 28, pp. 613646.

${ }^{22}$ B. Sturtevant, "Dynamics of vortices and shock waves in nonuniform media," Technical Report TR-79-0898, Air Force Office of Scientific Research, 1979. Available from Defense Technical Information Service, Govt. Accession No. AD-A098111, Microfiche N80-15366.

${ }^{23}$ D. Pullin, "Vortex ring formation at tube and orifice openings," Phys. Fluids 22, 401 (1979)

${ }^{24}$ B. Sturtevant, "Dynamics of turbulent vortex rings," Technical Report TR-81-0400, Air Force Office of Scientific Research (1981), Available from Defense Technical Information Service, Govt. Accession No. ADA072842, Microfiche N81-24027.

${ }^{25}$ S. Widnall, D. Bliss, and C.-Y. Tsai, "The instability of short waves on a vortex ring,” J. Fluid Mech. 66, 35 (1974).

${ }^{26}$ T. Maxworthy, "Some experimental studies of vortex rings," J. Fluid Mech. 81, 465 (1977).

${ }^{27}$ E. Broadbent and D. Moore, "Acoustic destabilization of vortices," Philos. Trans. R. Soc. London 290, 353 (1979).

${ }^{28}$ S. Widnall and C.-Y. Tsai, "The instability of the thin vortex ring of constant vorticity," Philos. Trans. R. Soc. London 287, 273 (1977).

${ }^{29} \mathrm{~T}$. Maxworthy, "Turbulent vortex rings (with an appendix on an extended theory of laminar vortex rings)," J. Fluid Mech. 64, 227 (1974).

${ }^{30} \mathrm{P}$. Saffman, "The approach of a vortex pair to a plane surface in inviscid fluid,” J. Fluid Mech. 92, 497 (1979).

${ }^{31} \mathrm{~W}$. Morton, "On the displacements of the particles and their paths in some cases of two-dimensional motion of a frictionless liquid," Proc. R. Soc. London, Ser. A 89, 106 (1913).

${ }^{32}$ A. Love, "On the stability of certain vortex motions," Proc. London Math. Soc. 25, 18 (1893).

${ }^{33}$ F. Dyson, "The potential of an anchor ring-Part II," Philos. Trans. R. Soc. London 184, 1041 (1893).

${ }^{34}$ T. Maxworthy, "Comments on 'Preliminary study of mutual slip-through of a pair of vortices'," Phys. Fluids 22, 200 (1979).

${ }^{35}$ S. Stanaway, B. Cantwell, and P. Spalart, "A numerical study of viscous 
vortex rings using a spectral method," TM 101004, NASA, 1988; also S. K. Stanaway, Ph.D. thesis, Stanford University, 1988.

${ }^{36} \mathrm{D}$. Sallet and R. Widmayer, "An experimental investigation of laminar and turbulent vortex rings in air," Z. Flugwiss. 22, 207 (1974).
${ }^{37}$ S. Shadden, J. Dabiri, and J. Marsden, "Lagrangian analysis of fluid transport in empirical vortex ring flows," Phys. Fluids 18, 047105 (2006).

${ }^{38}$ The qualifier "generalized" is needed to include cases where A or B do not possess a linearly independent set of eigenvectors. 